\title{
El método Ferrán y la inmunización contra el cólera en una ciudad del Mediterráneo. Elche, agosto de 1891
}

The Ferrán method and the inmunization against cholera in a Mediterranean city. Elche, august 1891

\section{O método Ferrán e a imunização contra a cólera em uma cidade mediterrânea. Elche, Agosto de 1891}

\section{Mariano Monge Juárez ${ }^{1}$}

${ }^{1}$ Doctor en Historia Contemporánea por la Universidad de Alicante. Universidad de Murcia. Correo electrónico: mongejuarez@um.es

Cómo citar este artículo en edición digital: Monge Juárez, M. (2020). El método Ferrán y la inmunización contra el cólera en una ciudad del Mediterráneo. Elche, agosto de 1891. Cultura de los

Cuidados (Edición digital), 24 (56) Recuperado de http://dx.doi.org/10.14198/cuid.2020.56.09

Correspondencia: C/Mariano Benlliure 29, 7º-2, 03201 Elche (Alicante) Correo electrónico de contacto: mongejuarez@um.es

Recibido:04/11/2029

Aceptado:27/02/2020

\section{ABSTRACT}

Objective: to analyze the process of vaccination against cholera - the Ferrán method - that was experienced in Elche, during August and September 1891, immunization promoted by municipal doctors and by Jaime Ferrán himself, who had visited the city several times. Thus, after the serious epidemics of 1884 and 1885, Elche received mandatory vaccination shortly after the first practices carried out in
Alzira or Valencia, in full scientific and journalistic controversy over the discovery of the Catalan scientist. Methodology: For the analysis of the facts we have used several sources. And the proposed analysis has been used. Báguena (2011) on the "Ferrán method". Primary documents of the Municipal Historical Archive of Elche (AHME) have been analyzed. Results: The Ferrán method, or first vaccine against morbid cholera, was a controversial preventive measure against this epidemic disease in Spain, from its practical application in several municipalities of the Kingdom of Valencia we interpret the 
Revista científica de la Asociación de Historia y Antropología de los Cuidados (Universidad de Alicante)

political and social weight of the municipal power, against the poor practical operation of the central governments of the Restoration. Conclusions: Manuel Campello and Santiago Pomares, in full controversy of the Ferrán vaccine, decided to launch a massive and compulsory vaccination for the entire population of the city, paying special attention to the poor classes.

Keywords: cholera, epidemic, vaccine, Ferrán method, poor.

\section{RESUMEN}

Objetivo: analizar el proceso de la vacunación contra el cólera -método Ferránque se experimentó en Elche, durante agosto y septiembre de 1891, inmunización impulsada por los médicos municipales y por el propio Jaime Ferrán, que había visitado la ciudad varias veces. De este modo, tras las graves epidemias de $1884 \mathrm{y}$ 1885, Elche recibió la vacunación obligatoria poco después de las primeras prácticas efectuadas en Alzira o Valencia, en plena controversia científica y periodística sobre el descubrimiento del científico catalán. Metodología: Para el análisis de los hechos nos hemos servidos de varias fuentes. Y se ha empleado el análisis propuesto. Báguena (2011) sobre el "método Ferrán”. Se han analizado documentos primarios del el Archivo Histórico Municipal de Elche (AHME). Resultados:El método Ferrán, o primera vacuna contra el cólera morbo, fue una controvertida medida preventiva contra esta enfermedad epidémica en España, de su aplicación práctica en varios municipios del Reino de Valencia interpretamos el peso político y social del poder municipal, frente a la escasa operatividad práctica de los gobiernos centrales de la Restauración. Conclusiones: Manuel Campello y Santiago Pomares, en plena controversia de la vacuna Ferrán, decidieron poner en marcha una vacunación masiva y obligatoria para toda la población de la ciudad, prestando especial atención a las clases pobres.
Palabras clave: cólera, epidemia, vacuna, método Ferrán, pobres.

\section{RESUMO}

Objetivo: analisar o processo de vacinação contra a cólera - método de Ferrán - com o qual foi vivida em Elche nos meses de agosto e setembro de 1891, imunização promovida pelos médicos municipais e pelo próprio Jaime Ferrán, que havia visitado a cidade várias vezes. Assim, após as graves epidemias de 1884 e 1885, recebeu a vacinação obrigatória após as primeiras práticas realizadas em Alzira Valencia, em plena controvérsia científica e periódica sobre a descoberta da ciência catalã. Metodologia: Para a análise dos tempos, usamos várias fontes. As análises propostas foram realizadas. Báguena (2011) sobre o "Método Ferrán". Os documentos primários foram analisados pelo Arquivo Histórico Municipal de Elche (AHME). Resultados: O método Ferrán, o primeiro caso contra a cólera mórbida, foi uma medida preventiva controversa contra essa epidemia na Espanha, sua aplicação prática em vários municípios do Reino de Valência interpretou o peso político e social do poder municipal à sua frente. Pouca operação prática dos governos centrais da Restauração. Conclusões: Manuel Campello e Santiago Orchards, no meio da controvérsia de vagas de Ferrán, decidiram lançar uma vacinação maciça e obrigatória para toda a população da cidade, prestando atenção especial às classes pobres.

Palavras chave: cólera, epidemia, vacina, método de Ferrán, pobre.

\section{INTRODUCCIÓN}

El contexto: la vacuna de Ferrán y el emergente poder municipal en España

Los últimos lustros del siglo XIX en Europa y España se caracterizan por un progreso 
económico y científico que nunca antes se había experimentado. Las ciudades se refundan, desde nuevos paradigmas urbanísticos y arquitectónicos, se liberan de murallas y calles insalubres. El Estado y las administraciones provinciales y municipales avanzan a marchas forzadas en un proceso en el que la sociedad civil se construye y se empodera como palanca de cambio social.

En este contexto, la lucha contra la amenaza epidémica significa uno de los ámbitos en los que dicha sociedad civil desarrolla sus funciones con especial intensidad. El cólera morbo asiático había acentuado su presencia en el mundo a lo largo de seis grandes ciclos pandémicos: desde las primeras décadas del siglo XIX, hasta 1891, momento en que se registra el último gran ciclo en toda Europa. Mientras los ferrocarriles y la electricidad comunican lejanos rincones de Europa y América, el ominoso espectro del cólera compite con el progreso y el optimismo decimonónico, y cada año cobra cientos de miles de víctimas anónimas que diezman la población activa, dañan el consumo y dejan en serios aprietos las estructuras productivas industriales $\mathrm{y}$ agrarias del capitalismo emergente. Filósofos tan influyentes como J. G. Fichte (1762-1814) o G. W. F. Hegel (1770-1831), artistas como J. M. W. Turner (1775-1851), científicos como Georges Cuvier (17691932) o el caso paradójico del epidemiólogo Juan Bautista Peset i Vidal (1821-1885), pierden la vida en las grandes epidemias de Berlín, París, Valencia o Londres y simbolizan la debilidad de la flamante sociedad occidental frente la estocada infecciosa.

\section{DESARROLLO DEL TEMA}

Como en Europa o América, las ciudades españolas toman conciencia activa de la importancia de las políticas preventivas, primero mediante las ya conocidas estrategias de aislamiento y de mejora en la salud pública: las calles se pavimentan, las alcantarillas llegan a más lugares, se cambian trazados urbanos que pretenden una mayor ventilación pero, sobre todo, los municipios se ocupan de evitar aguas estancadas; en un segundo momento, los municipios ponen en marcha la aplicación de los avances científicos. De este modo en cuanto la prensa y las autoridades políticas tienen noticia de la posible existencia de una vacuna contra el cólera, se organizan campañas de vacunación masivas administradas por los ayuntamientos, tal es el caso de Alzira, en abril de 1885, dirigida por el mismo Jaime Ferrán (1851-1929), Amalio Gimeno (1852-1936) y el químico Inocencio Pulí, población a la que sucedieron, a los pocos días, Chiva, Cheste o la misma Valencia. No obstante, la vacuna de Ferrán choca en aquellos momentos con importantes detractores como Carlos María Cortezo (1850-1933), Luis Simarro (18511921) o el mismo Santiago Ramón y Cajal (1852-1934), entonces catedrático de la 
Revista científica de la Asociación de Historia y Antropología de los Cuidados (Universidad de Alicante)

Universidad de Valencia, que recibe con grandes dudas el descubrimiento del científico catalán. El método de Ferrán levanta una enconada controversia, cuyo resultado termina siendo la hostilidad social contra la vacuna, sobre todo tras el incidente del Asilo de la Hermandad de Pobres de Valencia, en el que Ferrán había vacunado un total de 88 personas, entre asilados y monjas, de las que resultarán infectadas 30 y fallecidas 16 .

La consecuencia es que el médico catalán se negó a seguir con las campañas de vacunación locales y publicó un manifiesto en el que daba sus razones para suspender las inmunizaciones. Poco antes, todavía se había alcanzado a vacunar en los municipios de Ondara, Cambrils y Santa Pola (Báguena, 2011:4-6).

Nos encontramos, por tanto, ante la llamada “cuestión Ferrán”, en la que se ponía de manifiesto el conflicto entre una parte de la comunidad científica y la imperiosa necesidad de poderes locales frente a la constante amenaza de la epidemia. Y, ante la falta de consenso y arbitraje por parte del gobierno de la Restauración, fueron las estructuras municipales y provinciales las que desarrollaron una estrategia organizativa, al margen de las controversias científicas. El Ayuntamiento de Linares y las Diputaciones de Granada, Sevilla, Albacete, Córdoba o Logroño (Matilla, 1977) nombraron unas comisiones que pretendían estudiar el método de inoculación de Ferrán y el caso de Valencia. El primer obstáculo con el que se encontró la comisión fue la memoria sobre la epidemia de 1885 que había redactado la Junta Municipal de Sanidad del Ayuntamiento de Valencia (Bonachia, 2015), decididamente contraria a la vacuna de Ferrán, en la que, a lo largo de sus 248 páginas, eran numerosos los argumentos y las pruebas con las que la Junta desaconsejaba la vacuna:

“[el] período más alto de la epidemia, ha coincidido con el tiempo en que las vacunaciones fueron consentidas y que el crecimiento de la enfermedad, coincidió también con esta época, en la cual fuimos perdiendo la filiación de los casos que hasta entonces habíamos seguido con regularidad” (JMS, Valencia,1885).

Por el contrario, la comisión destacada en Valencia extrajo una conjetura muy distinta, que:

"la doctrina en que se apoya el método preventivo contra el cólera es racional y científica, y que si bien los experimentos hasta ahora practicados no permiten asegurar en absoluto que se ha descubierto la verdadera vacuna del cólera, los resultados practicados ya conocidos y bastante numerosos, permiten abrigar la fundada esperanza de que el Doctor Ferrán podrá en breve ser considera como el autor de ella” (Bonachia, 2015).

Es decir, “la cuestión Ferrán” emprendía el camino de ser superada, y el 
vacunólogo de Tortosa estaba a punto de pasar a la historia de la medicina como el primer bacteriólogo que, en palabras de Paul Ehrich, había conseguido "la inmunización activa del hombre frente a las bacterias de una forma admirable y con un objetivo determinado” (López Piñero, 2006).

En definitiva, la primera conclusión general que podemos extraer de este trabajo nos indica que fueron las administraciones municipales y provinciales y los servicios sanitarios locales los que desarrollaron las principales acciones preventivas e inmunizadoras contra el cólera u otras enfermedades epidémicas durante las últimas décadas del siglo XIX. El caso de la vacuna de Ferrán en Elche supone, desde la historiografía actual, un hecho paradigmático de intervención, en el que se observa cómo los municipios se ocuparon y preocuparon de proteger a su ciudadanía del modo más eficaz.

\section{La Junta Municipal de Sanidad en Elche y la vacunación de 1891}

Los servicios sanitarios de carácter público de una ciudad eran aquellos que dependían de la iniciativa o el mantenimiento del Ayuntamiento. Los médicos municipales recibían un salario que salía de las arcas públicas, cuya principal fuente de ingreso eran los impuestos sobre la propiedad urbana -amillaramientos- y los consumos. Estos médicos municipales solían ser profesionales de prestigio

profesional y moral, y constituían una de las oligarquías más influyenten de la ciudad. En el caso de Elche, es conveniente destacar en este sentido la figura del Doctor Manuel Campello Antón (1833-1904) y de Santiago

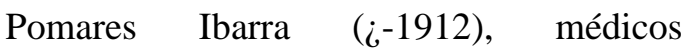
municipales ambos.

Durante la última década del siglo XIX, los servicios sanitarios se encontraban determinados por dos grandes procesos históricos: por una parte, el permanente estado de alarma epidémica que vivía la ciudad desde la "hecatombe de 1854", y por otra, el gran desarrollo que tuvo que experimentar la beneficencia en plena expansión de la industria local.

La encrucijada de documentos relacionados con el "huésped del Ganges" es tal que resulta difícil distinguir los aspectos estructurales de los coyunturales en materia de servicios sanitarios, ya que, en los últimos quince años del siglo XIX, la presencia de la epidemia había sido tal, que había producido toda una respuesta estructural en el campo médico y de la salud pública, que superó, con mucho, las posibles coyunturas relacionadas con el momento agudo de infección. Unido a esto, nos encontramos con una pobreza orgánica, imbricada en la nueva sociedad ilicitana y ante la cual el municipio respondía con la organización de una beneficencia basada precisamente en la asistencia sanitaria y farmacológica, sobre todo, porque la miseria suponía un foco humano de infección abierto y peligroso 
Revista científica de la Asociación de Historia y Antropología de los Cuidados (Universidad de Alicante)

para el resto de la población, incluidas las clases dominantes.

Como ocurrió en otros núcleos industriales europeos, el avance de la sociedad fabril, y sobre todo de la proletarización de las nuevas clases sociales, fue la causa de considerables desigualdades y bolsas de pobreza urbana. Elche vivió este proceso, proceso sin el cual sería imposible un análisis de los servicios sanitarios de la ciudad, ya que los pobres formaron una parte elemental de la política sanitaria $y$, obviamente, en lo concerniente a la beneficencia que desarrolló el municipio desde 1884 hasta los primeros años del siglo XX.

De entre las comisiones permanentes municipales, la de Beneficencia y la de Sanidad fueron las más activas, ya que asumieron la responsabilidad de dar respuesta a las epidemias o inundaciones, males endémicos en el área mediterránea. Basta un vistazo a los Libros de Actas Municipales (LAM) para advertir la actividad de dichas comisiones, que casi a diario, recibían, bien solicitudes de vecinos que pedían ser inscritos en la lista de pobres con el objeto de ser atendidos por un médico y recibir un tratamiento, bien solicitudes de lactancia de jornaleros que habían enviudado, y cuyos sueldos no daban para pagar los servicios de una nodriza.

La Junta Municipal de Sanidad (JMS) se componía de un médico, un cirujano, un farmacéutico, un veterinario y tres vecinos. Dicha Junta coordinaba las políticas sanitarias de la ciudad, tales como estrategias preventivas en cuanto a valoración de focos de infección -urbanos o rurales-, reparto y control de cartillas sanitarias (JMS, 19-06-1890), actividades fabriles perjudiciales para la salud pública en el casco urbano (El Pueblo, 01-05-1891), vacunación contra la viruela (LAM, 05-041902) o el cólera, supervisión de la cárcel municipal (LAM, 26-06-1890), demanda de presupuestos (Sig. 39-33-7) y control sobre los fármacos administrados a los pacientes pobres -sobre todo contra el paludismo(Sig.4-31), coordinación de la asistencia médica a los pobres, así como también se ocupaba de dirigir los protocolos de intervención en caso de epidemia. La Junta también controlaba la convocatoria de plazas de médicos y farmacéuticos municipales. El número de médicos municipales oscilaba entre 5 y 7, según la división administrativa de la ciudad en distritos, lo cual equivalía a una ratio aproximadamente de unos 4.000 habitantes por médico, si estimamos la población media del periodo 1880-1900 en unos 25.000 empadronados en total. Por ejemplo, en 1902, con una población de algo más de 27.000 habitantes, se registran 7 patentes de cirujanos municipales (Sig. D107).

Otro servicio sanitario fue el de las comadronas o parteras municipales, que también recibían un salario anual del Ayuntamiento y que desempeñaban mujeres con experiencia. 
Por otra parte, en la ciudad existía un hospital y un asilo (Sig. D-83-1), ambas instituciones gestionas por el Ayuntamiento y la Iglesia. La nómina de empleados del hospital de la caridad que el Ayuntamiento tenía que afrontar cada año económico, estaba compuesta por un médico, un capellán, un enfermero, un barbero y un sacristán (Sig. D-91-1), el resto de personal eran monjas. Tanto el hospital como el asilo se ocupaban de la asistencia a los enfermos de la ciudad registrados en las "listas de pobres”. Para figurar en éstas era necesario estar empadronado en la ciudad, tener un informe de la comisión dirigida por un médico, y haber presentado la solicitud correspondiente en el Ayuntamiento.

No obstante, $\mathrm{y}$ al margen de los servicios sanitarios vinculados a la beneficencia municipal, desde los años noventa, la ciudad experimentó un incremento sensible del número de médicos que ejercían su profesión libremente, en consultas a las que acudían las clases sociales más pudientes, actividad de la que tenemos noticia a través de la prensa, gracias a la publicidad en anuncios (El Vinalopó, 15-03-1884) u otras referencias.

Lo mismo podemos decir de los avances médicos, de los que la ciudad estuvo al tanto, sobre todo en los relativos a la lucha contra el cólera, e incluso en cuanto a la controversia científica que mantuvieron Jaime Ferrán y Ramón y Cajal a mediados de la década de los ochenta. En marzo de 1885, Jaime Ferrán había comunicado sus descubrimientos bacteriológicos a la

Academia de Ciencia de París (Álvarez, 1946). Durante varias semanas, la prensa local ilicitana publicó una detallada "Memoria escrita por el eminentísimo Doctor Ferrán sobre la enfermedad colérica” (El Talismán, 16-04-1887), anticipo de lo que será el periodismo de divulgación científica durante el siglo XX.

El método de Ferrán había levantado grandes esperanzas en una ciudad que venía siendo castigada por el cólera desde las primeras décadas del siglo XIX. En plena “cuestión Ferrán”, el médico catalán había visitado la ciudad, probablemente a causa de su condición de masón y a su relación con otro médico también masón de gran prestigio social en Elche, Santiago Pomares Ibarra. En aquella visita, poco después de la primera vacunación masiva, la del 24 de abril de 1885 en Alzira, Jaime Ferrán exhibió el bacilus virgula al microscopio. Además, visitó el Huerto de Fenoll acompañado de la élite política y médica local, representada por Luis Gómez Aznar, director de sanidad de Santa Pola (El Vinalopó, 20-07-1884), y los ya citados Campello Antón y Pomares Ibarra (El Bou, 30-07-1885).

El periodo 1885-90 coincidió con una ausencia de cólera en la ciudad, pero la opinión pública y los políticos locales ya habían tomado conciencia de las dimensiones que la epidemia podía tener si se combinaba con los efectos sociales de desempleo y miseria que sufría la nueva y 
Revista científica de la Asociación de Historia y Antropología de los Cuidados (Universidad de Alicante)

emergente sociedad industrial. Fueron epidemia, en un foco de infección escasos los años de descanso. El cólera incontrolable, por ello la JMS desarrolló tres morbo volvió de nuevo a la ciudad en el verano de 1890. En aquel momento fue El labrador, dirigido por Juan de Mata Coquillat, el que dio la voz de alerta de la aparición de la epidemia en Rugat, un municipio de la Vall d'Albaida (Valencia). En este caso, el periodista incidía en las consecuencias económicas que podía desencadenar una nueva epidemia, sobre todo para la clase trabajadora,

"los necesitados, los obreros, no deben ni pueden ser abandonados, de ahí la razón que nos impulsa a dar el primer aviso a nuestras autoridades procurando despejarlo todo de la ficción y la mentira” (El Labrador, 22-061890).

Antes incluso de la aparición de infectados, la primera reacción del Ayuntamiento fue activar un nuevo protocolo de urgencia propuesto por el médico local, Alfredo Llopis (1861-1917), que había repartido una serie de cartillas sanitarias con las que se pretendía informar a la población y combatir los síntomas iniciales (LAM, 26-06-1890). Otra medida, ya habitual, fue la suspensión de las fiestas de la Virgen de la Asunción “con motivo de la proximidad del cólera en cercanos municipios” (LAM, 17-04-1890). Pero el principal objetivo del poder municipal era evitar que la masa de pobres se convierta en la "quinta columna" de la asistencia sanitaria y farmacológica para una población de pobres que hubo de estar regulada, controlada y definida en un padrón con nombres y apellidos; en segundo, potenció la presencia del hospital y el asilo, así como los socorros o auxilios para la lactancia de huérfanos, que ya era una constante en las funciones de la Junta de Beneficencia; y en tercer lugar, en 1891, el Ayuntamiento, bajo el control de los facultativos de la ciudad, decidió la vacunación gratuita contra el cólera de toda la clase pobre de la ciudad (El Eco Liberal, 25-10-1891), hecho que se extendió a la mayoría de la población con carácter obligatorio, puesto que los "no vacunados no podrán de manera alguna ser admitidos en escuelas, colegios” (LAM, 08-05-1890) del municipio. De modo que la vacunación se efectuó entre el 31 de agosto y el 1 de septiembre de 1891, en el espacio abierto de mayor envergadura de la ciudad, conocido como la Glorieta (El Eco Liberal, 06-091891), lugar en el que se fue inoculando, según el método de Ferrán, a la mayoría de la población ilicitana. El archivo municipal no ha conservado datos de vacunación, tampoco sabemos si en algún momento los hubo. Sólo disponemos de estadísticas relacionadas con los pobres de la ciudad de 1891, estadísticas que probablemente sirvieron para administrar la vacuna. Según la lista de pobres de la Comisión de 
Cultura de los Cuidados

Beneficencia Municipal que se había efectuado durante el año 1891, y según la cual, en Elche existían 517 familias pobres, es decir, unas 1.800 personas, aproximadamente un $14 \%$ de la población urbana, ya que, según estas estadísticas, el $98,8 \%$ de las citadas familias se encontraban empadronadas en el casco urbano, y casi el 70 \% se localizaba en el raval de Sant Joan y en el de Santa Teresa. Tanto el Ayuntamiento como las organizaciones sindicales, aglutinadas bajo el Circulo Obrero Ilicitano (COI) -que también disponía de médico-, intentaron luchar contra los efectos de la miseria. La Corporación pretendió mejorar su sistema de beneficencia fundamentado en las ayudas de lactancia y la asistencia sanitaria, sobre todo orientado, desde 1891, a la vacunación gratuita contra el cólera o la viruela mediante los socorros mutuos, cuyo fin era salvar las situaciones más agudas de las familias obreras que habían perdido su empleo.

\section{CONCLUSIONES}

El uso de la vacuna de Ferrán contra el cólera, única hasta ese momento, se puso en práctica en Elche, igual que en otras ciudades españolas, por iniciativa exclusivamente municipal, al margen de controversias. Es necesario ubicar el hecho histórico en la necesidad de los poderes municipales ante la falta de respuesta de los gobiernos de la Restauración.
La incidencia del cólera en las masas de población urbana empobrecida por los efectos de la industrialización, motiva especialmente a las élites políticas de la ciudad para poner en marcha la vacunación obligatoria contra el cólera, ya que la enfermedad disminuía la mano de obra y los pobres constituían un foco humano de infección que podía afectar a la salud de las clases dominantes, además de un probable factor de inestabilidad social.

La vacunación contra el cólera fue una parte de la estrategia que siguió la Junta Municipal de Sanidad en coordinación con la de Beneficencia para impedir que los pobres se convirtieran en la "quinta columna" de la epidemia en la ciudad, las otras dos fueron, por una parte, la asistencia sanitaria y farmacológica, por otra, la mejora del hospital y el auxilio de lactancia para huérfanos.

La relación personal entre Jaime Ferrán y el médico local Santiago Pomares, ambos miembros de la masonería, fue un elemento importante, primero en la relación de Ferrán con Elche, y posteriormente con la puesta en práctica de su método en plena controversia.

La vacunación se efectuó entre el 31 de agosto y el 1 de septiembre de 1891, en el mayor espacio abierto de la ciudad, la conocida "glorieta del doctor Campello". Es importante incidir en su carácter obligatorio, gratuito para “clase pobre” y se dirigió especialmente a la población en edad 
Revista científica de la Asociación de Historia y Antropología de los Cuidados (Universidad de Alicante)

escolar. No se conocen estadísticas ni resultados.

\section{FUENTES Y BIBLIOGRAFÍA}

\section{FUENTES}

\section{Prensa histórica}

El Bou. Noticias actuales. 30 de julio de 1885.

El Eco Liberal. Noticias nacionales. 6 de septiembre de 1891.

El Eco Liberal. Noticias nacionales. 25 de octubre de 1891.

El Labrador. El cólera y los obreros. 22 de junio de 1890.

El Pueblo. Noticias. 1 del 17 de mayo de 1891.

El Talismán. Memoria escrita por el eminentísimo Doctor Ferrán sobre la enfermedad colérica. 16 de abril de 1887.

El Vinalopó. Noticias. 15 de marzo de 1884.

El Vinalopó. Noticias. 20 de julio de 1884.

\section{Otros documentos}

Libro de Actas Municipal (LAM), sesión del 17 de abril de 1890. Archivo Histórico Municipal de Elche (AHME). Sig. a 181.

LAM, sesión del 8 de mayo de 1890. (AHME). Sig. a 181.

LAM, sesión del 26 de junio de 1890. (AHME). Sig. a 181.

LAM, sesión del 5 de abril de 1902. (AHME). Sig. a 193, folio 26. Sig. a 181. Junta Municipal de Sanidad Elche (JMS). Sesión del 19 de junio de 1890. (AHME).
Ayuntamiento de Valencia (1885).El cólera en la ciudad de Valencia en 1885: Memoria de los trabajos realizados durante la epidemia por la Alcaldía del Excelentísimo Ayuntamiento en nombre de la Junta Municipal de Sanidad. Valencia, Imp. Manuel Afuere.

Sig. 39-33-7. Solicitud del farmacéutico Manuel Pomares Ibarra para el incremento del presupuesto para medicinas dado el volumen de la lista de pobres. (AHME).

Sig. 4-31. Acta de la Junta de Beneficencia, 30 de agosto de 1889. (AHME).

Sig. D-107. Relación de médicos cirujanos que se han provisto de las patentes para el año actual, en correspondencia municipal de 1902. Archivo Histórico Municipal de Elche (AHME).

Sig. D-83-1. Según el interrogatorio de la Diputación Provincial de noviembre de 1887. (AHME).

Sig. D-91-1. Nómina de empleados del hospital de la caridad de Elche, en “Correspondencia” del año 1894. (AHME).

\section{BIBLIOGRAFÍA}

Álvarez Sierra, J. (1946). Doctor Ferrán. Madrid: Ed. Naciona.

Báguena, M.J. (2011). Jaime Ferrán y su papel en las epidemias de cólera de Valencia. Anales Real Academia de Medicina de la Comunitat Valenciana, 12,4-6.

Bonachia, F. (2015).Memoria higienista de La Rioja. Una visión de la cultura social y sanitaria en el siglo XIX. Logroño: Tesis doctoral de la Universidad de la Rioja.

López Piñero,J.M. (2006). Santiago Ramón y Cajal. Valencia: Universidad de Valencia.

Matilla, V. (1977). Ferrán y su obra. Madrid: Instituto de España. 


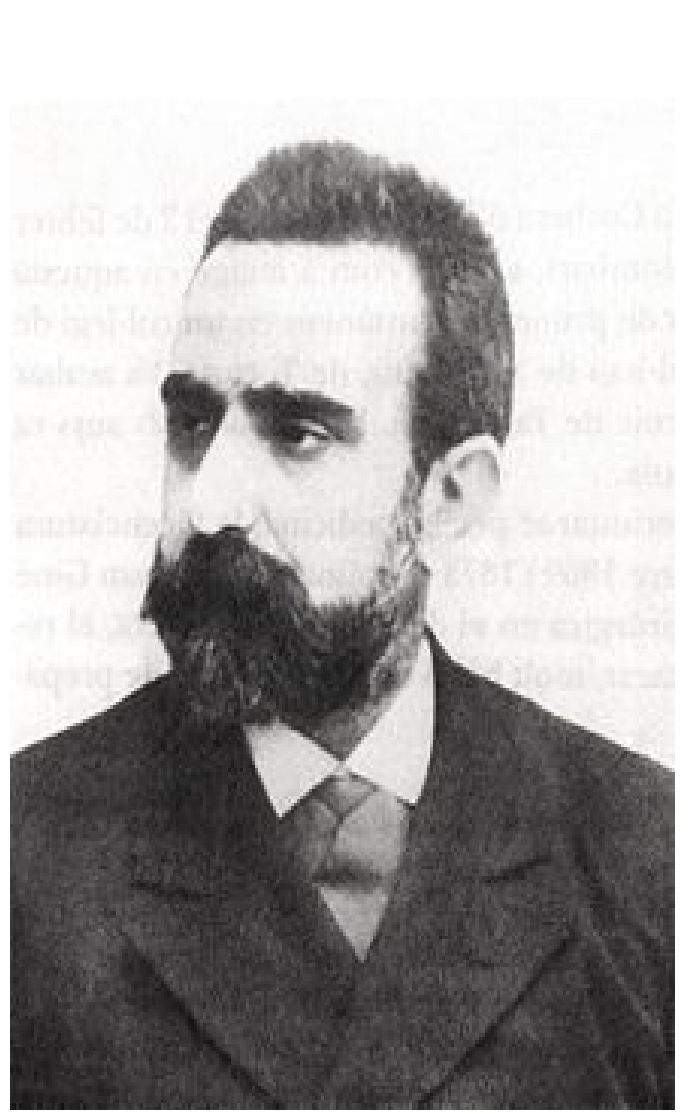

JAIME FERRÁN
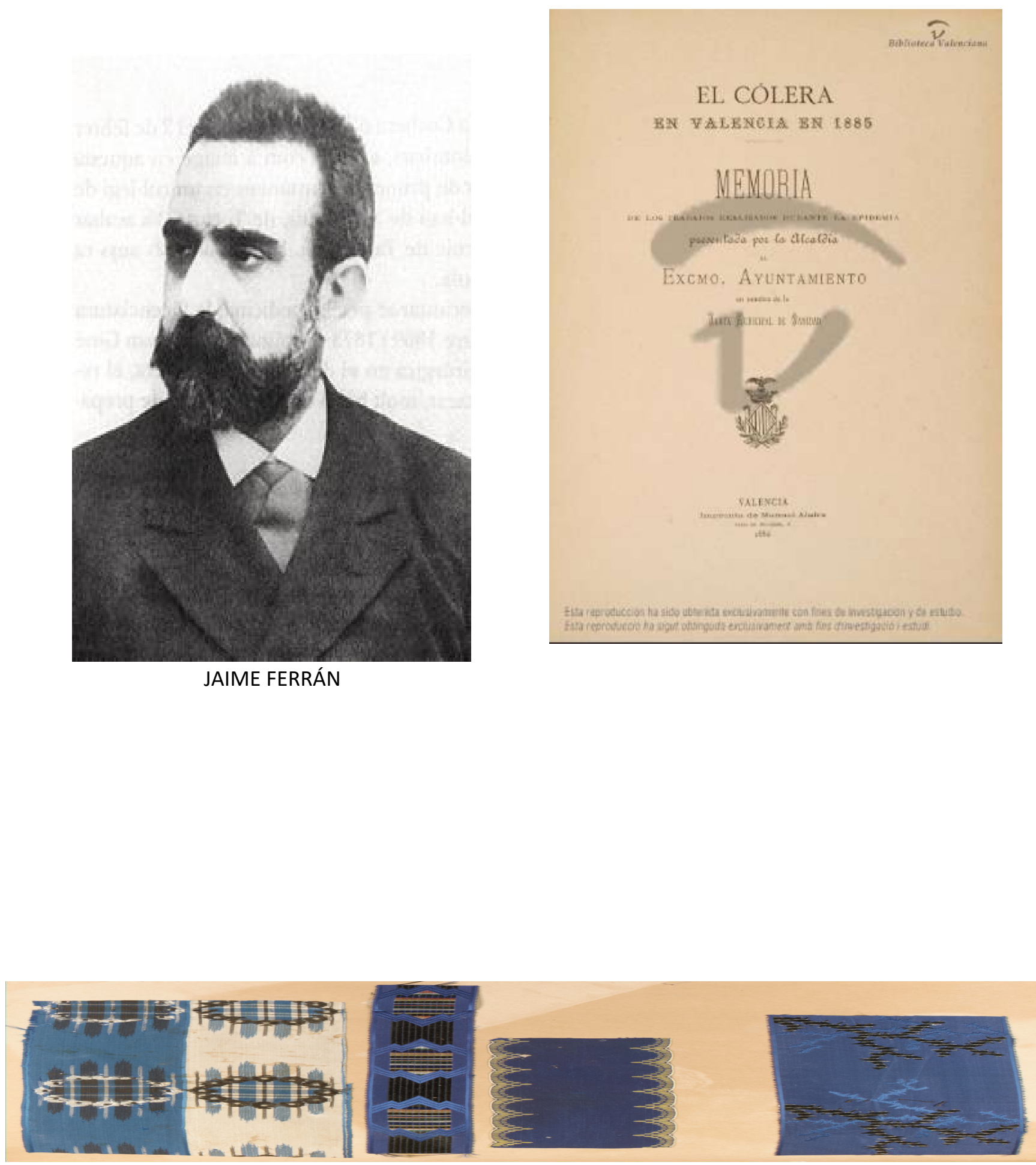

Fuente: https://www.metmuseum.org/art/collection/search/230928?

searchField=All\&amp;sortBy=AccessionNumberDesc\&amp;ao=on\&amp;ft=*\&amp;offset=160\&amp;rpp=40\&amp;pos=171 (Common Creative). 